\title{
Imaging single glycan molecules
}

\author{
Direct imaging of carbohydrates using electrospray ion beam deposition and scanning tunneling microscopy.
}

G lycans or carbohydrates are particularly challenging to characterize structurally. This is due to their complex and irregular branching patterns, highly flexible backbones, and different possibilities for stereochemistry at each glycosidic linkage. While direct imaging of various biomolecules has been feasible and routinely performed, structural characterization of glycans has relied on a combination of indirect measurements, such as mass spectrometry and nuclear magnetic resonance imaging. These methods provide an average measurement of structural features over multiple structures, or an ensemble. Direct imaging, by contrast, allows observation of characteristics of single isolated molecules.

Klaus Kern at the Max Planck Institute for Solid State Research, Stuttgart, Germany, and his former team member Stephan Rauschenbach, who now heads his own research group at Oxford, developed an approach using scanning tunneling microscopy (STM) in tandem with electrospray ion beam deposition (ES-IBD). The idea for isolating molecules by bringing them into the gas phase and then depositing them onto a surface for high-resolution imaging started with Rauschenbach's doctoral work with Kern, more than a decade ago, and Rauschenbach and Kern still work closely. Kern explains that the instrument that they built for the purpose "essentially brings the world of mass spectrometry, surface science and high-resolution imaging together."

Once you inject the sample, the instrument ionizes it, separates the components on the basis of mass and selectively brings the molecule of interest into an ultrahigh vacuum environment, reducing the pressure by 13 orders of magnitude. The approach also requires cryogenic temperatures to keep the molecules isolated by suppressing diffusion and conformational changes, before soft deposition on a surface devoid of impurities. This is followed by imaging using STM.

The researchers have previously imaged molecules including proteins, peptides and dyes, but extending the approach to glycans involved some unique challenges. They collaborated with the research group of Peter Seeberger, at the Max Planck Institute for

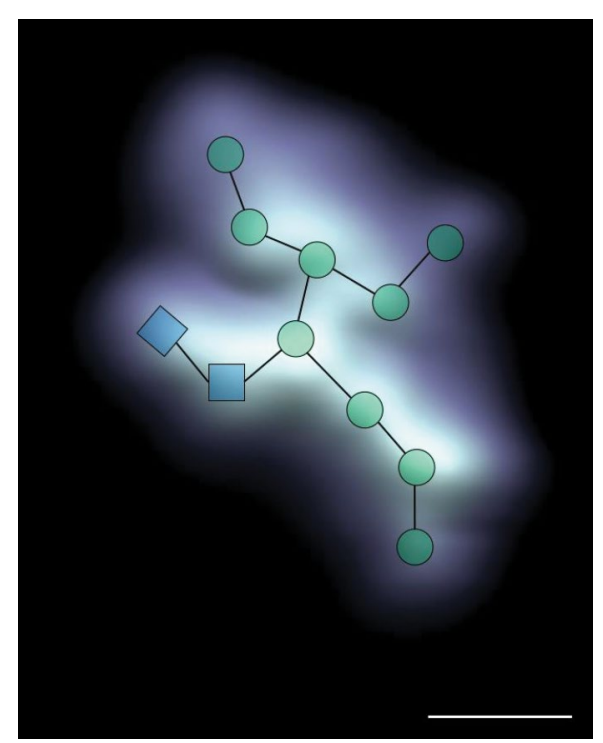

STM micrograph of a branched undecasaccharide consisting of nine mannoses (green circles) and two chitobioses (blue squares). Scale bar, $1 \mathrm{~nm}$. Courtesy X. Wu et al.

Colloids and Interfaces, Potsdam, Germany, which has mastered the carbohydrate synthesis process. Seeberger provided a variety of diverse glycans for these experiments, as well as the expertise needed to tailor the method to work with glycans. Electrospray ionization of glycans is done in negative mode, in contrast to the more common positive ion mode that is used for most other biomolecules like proteins and peptides, explains Rauschenbach. He also adds that glycans are not very interactive, so they used a 'sticky' copper surface, which tends to be more prone to contamination, also promoted by the low temperature used here. Given the sheer number of steps involved in the experimental setup, "it is crucial to hone every aspect of the process and make it work perfectly," says Rauschenbach.

In their recent publication, linear glycans with different lengths show up as distinctly observable protrusions, corresponding to the correct number of monosaccharide units, separated by distances that match known glycosidic bond lengths. The heights of various monosaccharides vary depending on the conformation adopted by them.
Isomers are also observed clearly, with unambiguous identification of the branching points. More complex glycans were also unmistakably identified (see image). "It was not clear if we would be able to see this. I was confident that we would see single molecules [rings] but we had no idea that we could even look at the bonding, so that we could even discriminate how they bond. So, for us it was really cool that we could identify these tiny differences," says Kern.

The setup is one of a kind - no other facilities are believed to exist at the moment that are capable of doing the exact same experiments. However, other groups are picking up the method. The driving force behind such collaborative multiscale projects is finding the right group of people. "You need someone who is interested in biology on one hand and in solid state physics on the other - a unique mix that you don't find everywhere," Rauschenbach points out. It took the researchers ten years to build and perfect the instrument, but as the applications get broader and the demand increases, there may be a possibility of commercialization.

In the meanwhile, a variety of more complex experiments are being performed and are planned for the future. The method is being employed to explore the conformational space of glycans by changing the collision energy before the deposition step, allowing molecules to adopt conformations of increasing energy. There is also the possibility of being able to extract specific glycans from a mixture using the mass-filtered deposition capability of ES-IBD. Another idea is to look at the interactions involving single glycan molecules directly, such as interactions with water or ions. "Glycans are interesting because there are a lot of questions that you cannot answer by any other method, but when we look at single glycans it allows us really to see conformations and detailed interactions," adds Kern.

\section{Arunima Singh}

Published online: 31 July 2020

https://doi.org/10.1038/s41592-020-0919-4

Research paper

$\mathrm{Wu}, \mathrm{X}$. et al. Imaging single glycans. Nature 582, 375-378 (2020). 